\title{
CULTURA CONSTRUCTIVA EN EL PROCESO DE URBANIZACIÓN DE LAS COMUNIDADES CAMPESINAS DEL VALLE DEL MANTARO
}

\section{BUILDING CULTURE IN THE PROCESS OF DEVELOPMENT OF RURAL COMMUNITIES MANTARO VALLEY}

\author{
Galván Canchanya Félix Alberto, Ccahuana Chocce Clemente
}

\section{RESUMEN.}

Para el estudio se estableció como unidades de análisis las comunidades de Cajas Chico, Huamanmarca y Azapampa, considerando que tuvieron procesos urbanísticos comparables que permite comprender estos procesos. Se plantearon los siguientes objetivos: a) Explicar el proceso de urbanización de las comunidades campesinas de Cajas Chico, Huamanmarca y Azapampa. b) Explicar las características de la cultura constructiva de estas poblaciones en el marco del proceso de urbanización que las afecta. Utilizamos el método histórico-analítico y además del método dialéctico que nos permitió ver el proceso evolutivo del crecimiento urbano (construcción de viviendas) en su efecto de servicio y el impacto de la migración. A partir del impacto de la globalización en los medios de comunicación, la difusión y adaptación al contexto de esa información, se puede comprobar a través del estudio que influye en la arquitectura de las comunidades de Cajas Chico, Azapampa y Huamanmarca a través de: su influencia económica, estilos de vida y estética. Estas se materializan a través de la redes de información, conocimiento; transferencias financieras internacionales, medios de comunicación masiva como la televisión, revistas, periódicos, etc. Y que son adaptadas en el contexto receptor. Los pobladores de las comunidades de estudio manifiestan su diversidad constructiva en el material, ornamentación, espacios y funciones de los mismos. Así, a través de la arquitectura de sus construcciones podemos analizar desde el punto de vista de la antropología sus actividades en espacios físicamente delimitados y que expresan significados simbólicos.

Palabras clave: Comunidades campesinas, proceso urbanístico, migración.

\section{ABSTRACT}

For this study the communities Cajas Chico, Azapampa and Huamanmarca were established as units of analysis, considering that they had comparable urban processes for the understanding of these processes. The following objectives were pursued: a) Explain the urbanization processes of the rural communities of Cajas Chico, Azapampa and Huamanmarca. b) Explain the characteristics of the constructive culture of these populations under the framework of the urbanization process that affects them. We use the historical-analytical method and also the dialectical method that allowed us to see the evolutionary process of urban growth (housing construction) in their effect on service and the migration impact. From the impact of globalization on the media, diffusion and adaptation to the context of that information, you can check through the study that it influences the architecture of the communities of Cajas Chico, Azapampa and Huamanmarca through: its economic influence, lifestyle and aesthetics. These are realized through information networks, knowledge; international financial transfers, mass media such as television, magazines, newspapers, etc. And they are adapted in the receiver context. The residents of the studied communities express their constructive diversity in the material, ornamentation, spaces and functions thereof. Thus, through the architecture of the buildings thier operations in confined spaces and physically expressing symbolic meanings can be analyzed from the point of view of anthropology.

Keywords: Rural communities urbanization process, migration. 


\section{INTRODUCCIÓN.}

Este trabajo tiene por finalidad exponer reflexiones sobre la cultura constructiva popular que ha creado un sistema informal de consolidación de las edificaciones para que éstas sean duraderas y puedan convertirse en patrimonio de varias generaciones. Las preguntas que fueron formuladas son: a) ¿Cuáles son los factores que dinamizan el proceso de urbanización de estas comunidades campesinas? y b) ¿Cuáles son las características de la cultura constructiva de estas poblaciones en el marco del proceso de urbanización que las afecta?

La cultura constructiva de la urbanización informal sigue produciendo y transformando de manera significativa el espacio urbano habitacional sin lograr los estándares de una vivienda y hábitat adecuado. Una primera reflexión surge de concebir el proceso de creación de la vivienda y el hábitat popular en las comunidades aledañas a los centros urbanos como parte de la dinámica cultural, entendida como un proceso de interacción que ocurre en un contexto social heterogéneo y desigual. Una segunda reflexión se dirige a como percibimos el proceso de consolidación de la vivienda en las comunidades. Una manera de mirar las formas culturales de los sectores populares ha sido a partir de sus relaciones de dependencia o condición subalterna respecto a la cultura dominante. Nuestra postura atiende al cambio de paradigma que reconoce en la acción de los hacedores de la comunidad su capacidad para producir cultura y nuevos significados de las prácticas relacionadas con la producción del hábitat y la vivienda.

Como hipótesis de trabajo se planteó que la construcción de viviendas de acuerdo al proceso de urbanización de la comunidades campesinas se deben a factores culturales, económicos y sociales, de acuerdo al grado de impacto de la cercanía con la urbe de Huancayo, a su dinámica migratoria y concepción del manejo de espacios de la vivienda de acuerdo al tipo de familia que la habita. Por ello el presente artículo muestra esta realidad, colocando su interés analítico en las condiciones socio - culturales del manejo y distribución de espacio, así como el tipo de vivienda construida. Se consideraron los siguientes objetivos: a) Explicar el proceso de urbanización de las comunidades campesinas de Cajas Chico, Huamanmarca y Azapampa y b) Explicar las características de la cultura constructiva de estas poblaciones en el marco del proceso de urbanización que las afecta.

\section{MATERIAL Y MÉTODOS}

\section{MATERIALES}

\section{a. Revisión bibliográfica} Objetivos:

- Enriquecer el Marco teórico del estudio.

- Explorar, como señalan los objetivos del planteamiento del problema, si existen referencias bibliográficas relacionadas con la interdisciplinariedad entre Antropología y Arquitectura.

\section{Actividades:}

- Se visitó la biblioteca para consultar diferentes libros relacionados con el tema, asimismo, se sacó copias de muchos de éstos a fin de profundizar su lectura. La revisión de los libros fue permanente durante toda la investigación.

\section{Instrumentos:}

- Fichas bibliográficas

- Biblioteca

\section{Materiales:}

- Libros de diversa índole

- Fichas

- Cuaderno de apuntes

- Lapiceros

\section{b. Observación} Objetivos:

- Conocer mejor el área de estudios, así como los focos de estudio.

- Analizar en el campo los cambios visibles en base al estereotipo moderno - rústico de las viviendas.

\section{Movimientos:}

- Se realizó un recorrido por el lugar de trabajo fotografiando todas las viviendas que se hallaron, en orden de las calles por donde se pasaba. Asimismo, nos detuvimos a ver las construcciones durante su realización así como descripción detallada.

\section{Instrumentos:}

- Guía de observación

\section{Materiales:}

- Cámara fotográfica

- Cuaderno de apuntes

- Diario de campo

- Lapiceros 


\section{c. Entrevistas:}

\section{Objetivos:}

- Detallar las razones de las construcciones de forma directa.

- Obtener información adicional

\section{Actividades:}

- Posteriormente al conteo, se seleccionó algunas viviendas para ser visitadas y entrevistar a los dueños 0 quienes estuvieron al tanto en el diseño de ésta; grabándose en video cada una de estas entrevistas. Luego se procedió al análisis interpretativo de los resultados.

\section{Instrumentos:}

- Guía de entrevistas

- Resultados del conteo

\section{Materiales:}

- Cuaderno de campo

- Cámara fotográfica (con videograbadora incluida)

- Diario de campo

- Lapiceros

\section{MÉTODO}

Utilizamos el método histórico-analítico y además del método dialéctico que nos permitió ver el proceso evolutivo del crecimiento urbano (construcción de viviendas) en su efecto de servicio y el impacto de la migración.

\section{RESULTADOS}

Ubicación y Extensión: En nuestro análisis nos referiremos a 3 comunidades: Cajas Chico, Azapampa y Huamanmarca.

A continuación presentamos las fotos satelitales que nos muestran sus ubicaciones en el Valle del Mantaro.

FOTO 1: Ubicación Geográfica de la CC.CC. Cajas Chico - Huancayo

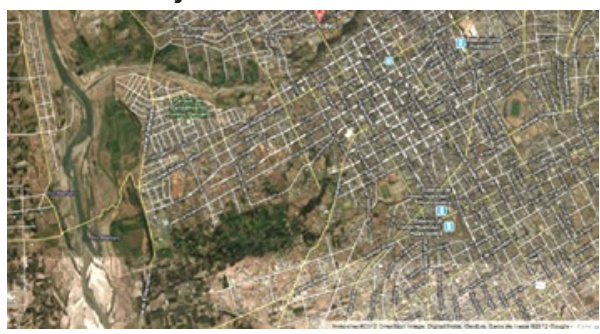

Fuente: Google Maps - 2010
FOTO 2: Ubicación Geográfica de la CC.CC.

\section{Azapampa - Chilca}

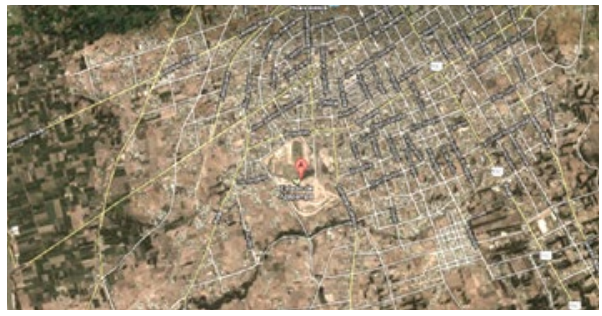

Fuente: Google Maps - 2010

FOTO 3: Ubicación Geográfica de la CC.CC. Huamanmarca

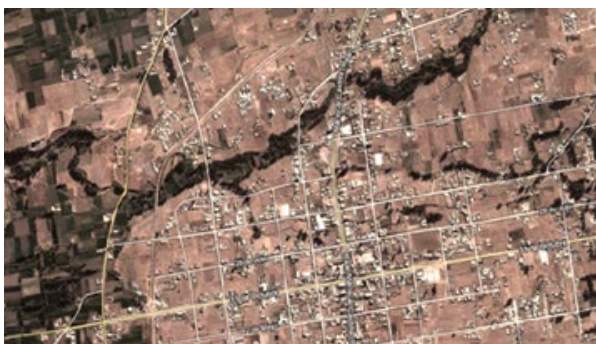

Fuente: Google Maps - 2010

Los estándares generales son modelos de cualificación que adoptan un grupo mayoritario y/0 están ampliamente difundidos; así, según el VI Censo Nacional de Viviendas pertenece al contexto rural, puesto que en ella los habitantes se dedican a actividades agropecuarias, en ese sentido su ordenamiento urbano pertenecería también a un esquema rural. La Municipalidad de Huancayo admite lo mismo para su jurisdicción. Sin embargo, este ordenamiento urbano está planeado de forma tal que en un futuro tenga características urbanas. Esto a nuestro entender, es parte de las Políticas de Desarrollo que consideran que la urbanización de lo rural (sobre todo de zonas cercanas a la ciudad) son indispensables para demostrar el progreso y/o nivel de desarrollo del conjunto provincial 0 distrital.

\section{CONTEO DE VIVIENDAS}

Se ha realizado un conteo de las viviendas de las comunidades de Cajas Chico, Azapampa y 
Huamanmarca. El conteo tiene como unidad a los edificios enteros, considerando en algunos casos la particularidad de su división o distribución (departamentos, cuartos de alquiler, etc.). De esta manera basándonos en las categorías utilizadas por el Instituto Nacional de estadística e Informática (INEI) en los censos, procedimos a clasificarlas.

En el caso de la comunidad de Cajas Chico se hizo el levantamiento de información de la calle Lima desde la cuadra 12 a la 16, y para hacer la comparación se consideró como referencia la calle Ica Nueva, desde Yauris hasta la calle Huancavelica. En el caso de la comunidad de Azapampa se consideró como referencia la calle Real y Túpac Amaru, CalleAmazonas, Calle Mendoza y Calle José Olaya. Finalmente en el caso de la comunidad de Huamanmarca se consideró la Av. Catalina Huanca y el perímetro de la Plaza Huamanmarca.

\section{CLASIFICACIÓN SEGÚN MATERIAL}

Se distinguen las viviendas de tres tipos de material: rústico, noble y mixto.

\section{Material Noble}

Son aquellas viviendas que fueron construidas en su mayor parte con material de concreto, es decir cemento ladrillos, estructuras metálicas etc. Dentro del discurso del desarrollo así como de la percepción de la población, estas viviendas representan un estilo de vida más cómodo y orientados a satisfacer las necesidades básicas y funcionales de sus habitantes. Además las posibilidades artísticas que conlleva las hacen aceptables para quienes poseen recursos; en nuestro trabajo la consideramos como fase final del proceso de cambio entre lo moderno y lo tradicional. Sin embargo a veces al dar prioridad a la estética no se toma en cuenta los perjuicios que conllevan, como la necesidad de calefacción a consecuencia de los materiales de concreto que usan en la altitud y clima de la zona.

\section{Material rústico}

Son viviendas que se caracterizan por estar construidas en su mayor parte con material rústico, de poca y/o sencilla elaboración como tapias, adobes, tejas, quinchos, piedras, etc. Consideramos a las viviendas con estas características como fase inicial del cambio en los estilos arquitectónicos debido a su sencillez y a la abundancia de éstas en la zona, su carácter rudimentario según el discurso del desarrollo; las etiquetamos como tradicionales. El desarrollo no las considera aptas para ser llevaderas de una vida digna para sus habitantes, por la dificultad en su limpieza y la instalación de servicios básicos necesarios para las personas que las habitan.

\section{Material mixto}

Viviendas construidas tanto con material noble como rústico. Muchas de ellas son ampliaciones, reparaciones o mejoras de un estado anterior generalmente rústico.

TABLA 1

\section{CLASIFICACIÓN SEGÚN MATERIAL Y PORCENTAJE}

\begin{tabular}{|l|c|c|c|c|c|}
\hline $\begin{array}{c}\text { Tipo de } \\
\text { material }\end{array}$ & $\begin{array}{c}\text { Cajas } \\
\text { chico }\end{array}$ & Huaman-marca & Azapampa & Total & $\%$ \\
\hline Mixto & 12 & 6 & 5 & 23 & $\mathbf{7 , 6 4}$ \\
\hline Noble & 71 & 18 & 22 & 111 & 36,88 \\
\hline Rústico & 66 & 56 & 45 & 167 & 55,48 \\
\hline Total & $\mathbf{1 4 9}$ & $\mathbf{8 0}$ & $\mathbf{7 2}$ & $\mathbf{3 0 1}$ & $\mathbf{1 0 0}$ \\
\hline
\end{tabular}

Fuente : Elaboración propia

\section{CLASIFICACIÓN SEGÚN TAMAÑO (ALTURA)}

Se distinguen diferentes tamaños que van desde uno hasta cinco pisos.

TABLA 2

\section{CLASIFICACIÓN ALTURA}

\begin{tabular}{|c|c|c|c|c|c|}
\hline Pisos & Cajas chico & Huamanmarca & Azapampa & Total & $\%$ \\
\hline 1 & 38 & 8 & 12 & 58 & 20,07 \\
\hline 2 & 53 & 26 & 10 & 89 & 30,80 \\
\hline 3 & 35 & 24 & 32 & 91 & 31,49 \\
\hline 4 & 20 & 10 & 18 & 48 & 16,61 \\
\hline 5 & 3 & 0 & 0 & 3 & 1,04 \\
\hline TOTAL & $\mathbf{1 4 9}$ & $\mathbf{6 8}$ & $\mathbf{7 2}$ & $\mathbf{2 8 9}$ & $\mathbf{1 0 0}$ \\
\hline
\end{tabular}

Fuente : Elaboración propia

\section{ESTILOS ARQUITECTÓNICOS}

El cambio de estilos arquitectónicos se nota más en los aspectos de ornamentación, y ampliación y adición de espacios para fines funcionales.

Estas nuevas tendencias de estilos se ven influidas por el desarrollo, las familias aspiran a una mejora en su calidad de vida, por ello diseñan viviendas con nuevos ambientes y más áreas funcionales.

Asimismo, estas nuevas tendencias son producto de la adopción de corrientes estilísticas, no quizá de la arquitectura contemporánea (consideradas como 'altas 
corrientes'), pero sí de populares que les permite hacer distinción sobre sus costumbres y situación económica. La adopción de corrientes se hace en alusión a la urbanización del lugar, donde imitan modelos de las urbes cercanas, en este caso la ciudad de Huancayo, y a su vez éstas de los modelos limeños, ello se da con mayor rapidez por la comunicación, acceso a la adquisición y las facilidades en el transporte. Además destaca la influencia económica de las remesas por parte de migrantes que envían a sus familiares en y la mejora económica de los dueños de las viviendas.

\section{AZAPAMPA}

FOTO 4: VIVIENDA DE MATERIAL RUSTICO

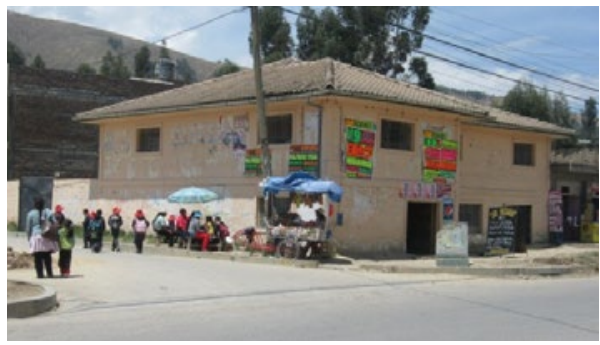

FUENTE: Elaboración propia, en base al trabajo de campo

En la foto podemos observar que este es una vivienda de material rustico, su localización en la esquina y entre dos avenidas se aprovecha por sus propietarios para cumplir las funciones de negocio en el segundo nivel las habitaciones, con cubierta de teja, ventanas pequeñas y puertas de madera.

\section{FOTO 5: VIVIENDA DE MATERIAL NOBLE}

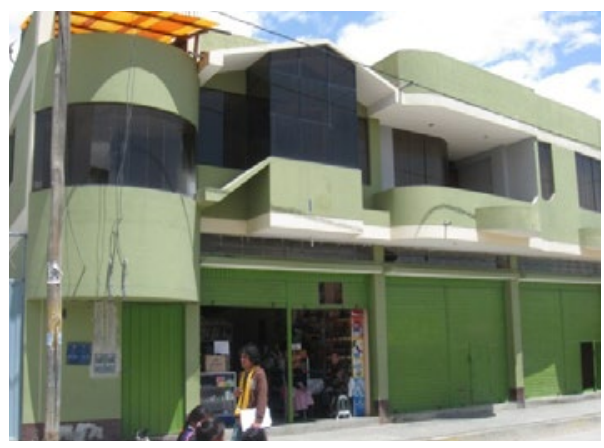

FUENTE: Elaboración propia, en base al trabajo de campo
Esta construcción es moderna, de material noble se aprovecha al máximo es espacio para que en la primera planta se vea hasta 3 puertas grandes dimensiones de fierro por la seguridad para utilizarla en los negocios, las ventanas son más amplias para aprovechar la luz del día y así ahorrar energía eléctrica, el acceso a los pisos superiores es través de una escales que tiene ingreso independiente.

\section{FOTO 6: VIVIENDA DE MATERIAL NOBLE}

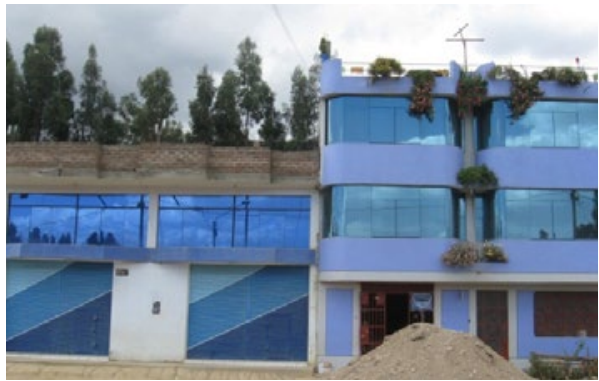

FUENTE: Elaboración propia, en base al trabajo de campo

La vivienda presenta también puertas amplias en el primer nivel para los negocios, el segundo y tercer nivel para habitaciones, lo particular es el color azul, con espejos reflejantes amplios, lo singular es que permanece la necesidad de incorporar plantas colgantes en la fachada.

\section{FOTO 7: VIVIENDA DE MATERIAL MIXTO}

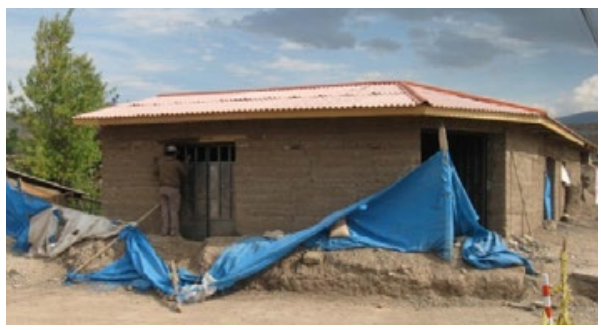

FUENTE: Elaboración propia, en base al trabajo de campo

Aun en este tiempo de globalización, este tipo de vivienda hace un referente a una vivienda con cubierta de material moderno, aunque el adobe mejorado es su principal material de construcción. 


\section{HUAMANMARCA}

FOTO 8: VIVIENDA DE MATERIAL RUSTICO

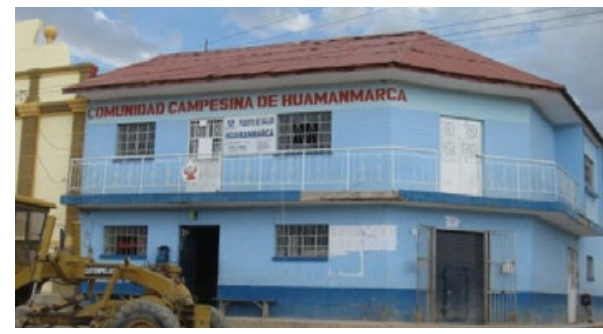

FUENTE: Elaboración propia, en base al trabajo de campo

Este inmueble se utiliza administrativamente, tiene algunas mejoras que fueron incorporándose en su afán de modernizarla y hacerla más funcionales, con materiales de fierro (cerrajería) se ve un balcón en el segundo nivel, la cubierta.

\section{FOTO 9: VIVIENDA DE MATERIAL NOBLE}

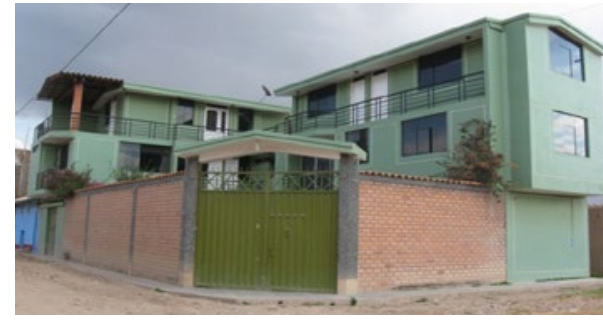

FUENTE: Elaboración propia, en base al trabajo de campo

Este inmueble se caracteriza por dejar un espacio al centro de la vivienda que se utiliza como un patio de servicio, se puede observar que en los dos niveles superiores se encuentra una balconera con fierro lo que representa una cultura constructiva en tránsito de manejar es espacio en viviendas rurales y urbanos.

\section{CAJAS CHICO}

FOTO 10: VIVIENDA DE MATERIAL RUSTICO

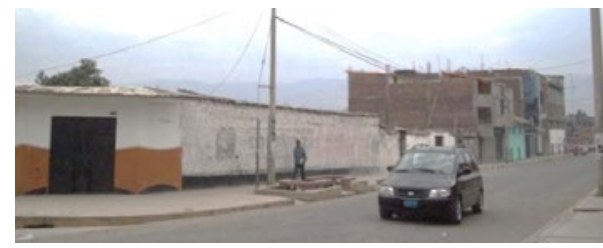

FUENTE: Elaboración propia, en base al trabajo de campo
Estas viviendas están se ubican cerca al Cementerio Central de Huancayo, por ello su función son generalmente para bares y tabernas. Se puede observar las viviendas de material noble de 3 pisos, están en proceso de urbanizarse.

\section{FOT0 11: VIVIENDA DE MATERIAL NOBLE}

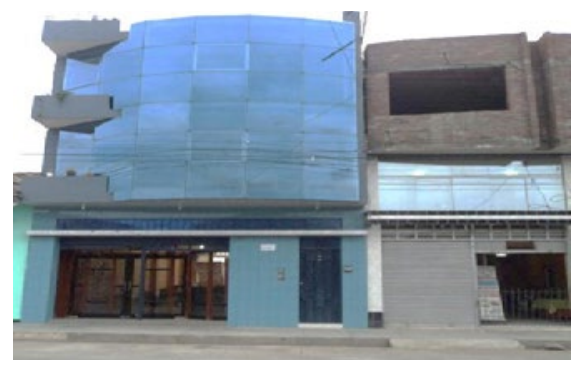

FUENTE: Elaboración propia, en base al trabajo de campo

En esta vivienda se puede observar la modernidad, es una imitación de edificios con vidrios reflejantes es un inmueble de 3 pisos pero hace esa emulación de los rascacielos, también se puede notar que la primera planta funciona como un negocios con una pequeña puerta que accede con una escalera reducida a los siguientes niveles, está en proceso de acabado y proyectado a más de 5 niveles

\section{DISCUSIÓN}

Primero vamos a abordar el carácter general del significado de Arquitectura, desde el punto de vista que los arquitectos y enciclopedistas, es decir el sentido común que tienen de ella; posteriormente la abordaremos desde la perspectiva antropológica, categorizándola como parte de un sistema cultural.

"La arquitectura abarca la consideración de todo el ambiente físico que rodea la vida humana: no podemos sustraernos a ella mientras formemos parte de la civilización."1

Wlliam Morris manifiesta que la Arquitectura es el conjunto de modificaciones y alteraciones introducidas en la superficie terrestre con objeto de satisfacer las necesidades humanas, exceptuando sólo el puro desierto.

1 MORRIS, William: "The Prospects of architecture in Civilization" en On Art and Socialism, Londres, 1947. Tomado de Wikipedia, referencia : Arquitectura 
"La arquitectura abarca la consideración de todo el ambiente físico que rodea la vida humana: no podemos sustraernos a ella mientras formemos parte de la civilización."

Tanto en la antigüedad como en los tiempos modernos arquitectura es considerada como un arte de edificar, con ello también entendemos que se necesita un estudio previo: una capacitación para desarrollar y optimizar la habilidad de diseñar construcciones, estos estudios en la era moderna se imparten en universidades, escuelas profesionales y otros equivalentes. La arquitectura como disciplina de las ingenierías (0 de las ciencias que se dedican a la construcción de inmuebles) es un sistema cultural, ya que es una forma de planificar y diseñar construcciones, entre las muchas otras formas que existen en la diversidad de culturas del mundo.

En la Antropología llamamos a la arquitectura "Formas Constructivas" porque existen muchas otras formas más de diseñar la construcción de edificios y viviendas, por lo cual la Arquitectura es uno más. Las formas constructivas son funcionales por varias razones, de los que destacan su aspecto como tecnología. Las construcciones son un bien de distinción y prestigio a través del significado de sus símbolos que se expresan en la forma, tamaño, material usado y ornamentos en las viviendas; todas las familias buscan distinguirse y perpetuar su orden en el campo social.

Bourdieu plantea las razones de distinción, teoría que explica que los hechos sociales no son absolutos, no son el motor de la conducta humana. Con ello podemos postular que la distinción no fue el motor absoluto del fenómeno de construcción de viviendas con estilo moderno, formaba parte de los motivos, pero no el motivo determinante. Además las personas negaran y en otros casos afirmaran cualquier signo de distinción puesto que su pertenencia al grupo social (la comunidad) depende de su grado de cohesión, pero que por el fenómeno de la globalización manifestaran su distinción en contextos diferentes.

Por ello se debe plantear para la comprensión y discusión de los procesos de reproducción y transformación del espacio urbano residencial auto producido y del contexto de la construcción de la ciudad moderna con sus marcadas desigualdades sociales.

2 Idem.
El estudio de la Arquitectura como rasgo cultural es reciente, según Denise Lawrence éste fue poco estimado por las primeras generaciones de antropólogos hasta que se preguntaron por la importancia del espacio y el tiempo en la teoría social: "Aunque los enfoques arquitectónicos utilizan numerosos conceptos culturales, los antropólogos pocas veces han investigado las formas constructivas en sí mismas. El reciente interés antropológico por la Arquitectura y por la parte material de la cultura, se debe a los esfuerzos por reubicar espacialmente (y también temporalmente), y preguntarse qué relación existe entre los seres humanos y las formas que construyen. Los estudios antropológicos tempranos veían a las formas constructivas como parte integral de la vida social y como elemento universal de la cultura, pero en general les daban un carácter pasivo en las descripciones etnográficas que sirvieron de entorno 0 de evidencias para diversos enfoques teóricos. "3

\section{ESPACIO FUNCIONAL}

Michel Foucault plantea que el espacio funcional se transforma por influencia de la globalización: la ampliación y la adición de estos. El fin por los que se dan es variado. Para la mejorar económica, la optimización de la reproducción social y distinción. Muchas viviendas han sido construidas con un fin funcional: tiendas, almacenes, 0 algún tipo de negocio. Esto se da debido a la expansión del capitalismo, si bien no es reciente, se está intensificando. Poco a poco la gente, influida por la idealización sobre la calidad de vida urbana, diseña la construcción de sus viviendas con los nuevos espacios ya señalados a fin de invertir en generadores de recursos económicos en mediano o largo plazo. Otro factor funcionalista es la ampliación del espacio para la optimación de la reproducción social. Encontramos casos de viviendas construidas con el fin de hacer que las familias de los diseñadores/constructores habiten cómodamente la vivienda en un corto o mediano plazo, y así mantener la continuidad de apoyo mutuo y estabilidad económica; para ello realizan estrategias de reproducción social (Bourdieu) en este caso la construcción de viviendas más amplias y más cómodas.

3 LAWRENCE, Denisse: "Arquitectura” en BARFIELD, Thomas (ed.): "Diccionario de Antropología". Siglo XXI, México 2000 p. 80 
Teófilo Altamirano piensa sobre la inversión de las remesas en construcción de viviendas cercana a la ciudad o en ella misma; si bien es cierto, también se hace necesario manejar otra teoría que nos dé razón sobre el por qué algunos beneficiados por las remesas mejoran económicamente y se quedan continuando su vida campesina y con ello, las mejoras en sus viviendas relacionadas con la continuidad de la práctica agropecuaria, como construcción de corrales, almacenes, etc. Pues bien, la reproducción cultural de Bourdieu, sostiene también que ciertas prácticas en áreas sociales (rurales 0 urbanas) producen distinción. Pero ésta distinción sólo se da dentro de su campo, es decir, dentro de los niveles culturales; así la gente que se dedica a actividades económicas similares trata de distinguirse entre ellos, fuera de ese contexto no puede hacerlo.

\section{IDENTIDAD Y ORNAMENTACIÓN}

Otro de los elementos que dinamizan estos cambios es la falta de estímulos económicos y culturales que permitan la migración a la ciudad (falta de dinero, de conocimiento del lugar de destino, reducida red social, etc.), Las costumbres muy arraigadas con el campo por parte de los pobladores. Una de las razones por la cual la gente recurre a formas similares para edificar sus viviendas es debido a su tangibilidad y perceptibilidad, son portadores de distinción. Distinción en muchos sentidos, en el cual nos enfocamos en la identidad. Según Pierre Bourdieu, la distinción es principio de la identidad. La gente trata de diferenciarse una de la otra usando signos similares a unos y diferentes a otros. Estos signos pueden encontrase en la estética.

\section{CONCLUSIONES}

1. A partir del impacto de la globalización en los medios de comunicación y la difusión y adaptación al contexto de esa información, se puede comprobar a través del estudio que influye en la arquitectura de las comunidades de Cajas Chico, Azapampa y Huamanmarca a través de: su influencia económica, estilos de vida y estética. Estas se materializan a través delaredes de información, conocimiento; transferencias financieras internacionales, medios de comunicación masiva como la televisión, revistas, periódicos, etc. $Y$ que son adaptadas en el contexto receptor.
2. El cambio de estilos arquitectónicos, tienen como fase inicial las viviendas rurales a las que consideramos "tradicionales", y como fase final a las viviendas de material noble que consideramos "modernas", son estas las cuales se expresan con mayor contundencia.

3. La arquitectura es una manifestación de la cultura a través de símbolos y posee aspectos funcionalistas, esto se pudo comprobar de acuerdo al sistema cultural, pues como otras expresiones de la cultura esta se planifican los tipos de construcciones que muestran la diversidad de cultural. Así, la arquitectura desde el punto de vista de la antropología consiste en las dimensiones materiales de la cultura que los seres humanos construyen para crear refugios, encerrar actividades en espacios físicamente delimitados y expresar significados simbólicos. A través interaccionismos simbólico se puede observar las diversas manifestaciones de las viviendas modernas las cuales se pueden clasificar en por dimensiones, formas, espacios y ornamentos, que expresan el prestigio de estas poblaciones que se modifican de acuerdo a las necesidades de espacios funcionales, como nuevas formas de manifestar su identidad local dentro de un espacio global.

\section{REFERENCIAS BIBLIOGRÁFICAS}

- AltamiRANO RUA, Teófilo. (2002) "El Trabajo de la Representación" en INSTITUTO DE ESTUDIOS PERUANOS: Taller: Prácticas y representaciones de la nación y ciudadanía en el Perú. Lima

- AltAmiRANO RUA, Teófilo. (2006) Remesas y Nueva "Fuga de Cerebros". Impactos transnacionales. Fondo editorial PUCP. Lima.

- BOURDIEU, Pierre. (1997) "Razones Prácticas para una Teoría de la Acción" Anagrama.

- BOURDIEU, Pierre (1988) La Distinción Criterio y bases sociales del Gusto. Alfaguara - Taurus. Madrid - España.

- LAWRENCE, Denisse. (2000). "Arquitectura" en BARFIELD, Thomas (ed.): Diccionario de Antropología. Siglo XXI, México 2000

- MORRIS, William. (1947) "The Prospects of architecture in Civilization" en On Art and Socialism. Londres. Tomado deWIKIPEDIA: referencia: Arquitectura. 